\title{
Recombinant Klotho Protects Human Periodontal Ligament Stem Cells by Regulating Mitochondrial Function and the Antioxidant System during $\mathrm{H}_{2} \mathrm{O}_{2}$-Induced Oxidative Stress
}

\author{
Huan Chen $\mathbb{D}^{\mathbb{D}}$, Xiaojun Huang $\mathbb{D}$, Chuanqiang Fu $\mathbb{D}$, Xiayi Wu, Yingying Peng $\mathbb{D}$, \\ Xuefeng Lin $\odot$, and Yan Wang \\ Guanghua School of Stomatology, Hospital of Stomatology, Sun Yat-sen University, Guangdong Provincial Key Laboratory \\ of Stomatology, Guangzhou 510055, China \\ Correspondence should be addressed to Xuefeng Lin; linxfeng@mail.sysu.edu.cn and Yan Wang; wang93@mail.sysu.edu.cn
}

Received 27 August 2019; Accepted 29 October 2019; Published 28 November 2019

Academic Editor: Ana Cipak Gasparovic

Copyright (c) 2019 Huan Chen et al. This is an open access article distributed under the Creative Commons Attribution License, which permits unrestricted use, distribution, and reproduction in any medium, provided the original work is properly cited.

\begin{abstract}
Human periodontal ligament stem cells (hPDLSCs) are a favourable source for tissue engineering, but oxidative stress conditions during cell culture and transplantation could affect stem cell viability and stemness, leading to failed regeneration. The aim of this study was to evaluate the antioxidant and protective effects of Klotho, an antiageing protein, against cell damage and the loss of osteogenesis in hPDLSCs in $\mathrm{H}_{2} \mathrm{O}_{2}$-induced oxidative environments. $\mathrm{H}_{2} \mathrm{O}_{2}$ was used as an exogenous reactive oxygen species (ROS) to induce oxidative stress. Recombinant human Klotho protein was administered before $\mathrm{H}_{2} \mathrm{O}_{2}$ treatment. Multitechniques were used to assess antioxidant activity, cell damage, and osteogenic ability of hPDLSCs in oxidative stress and the effects of Klotho on hPDLSCs. Mitochondrial function was analyzed by an electron microscopy scan of cellular structure, mitochondrial DNA copy number, and cellular oxygen consumption rate (OCR). Furthermore, we explored the pathway by which Klotho may function to regulate the antioxidant system. We found that pretreatment with recombinant human Klotho protein could enhance SOD activity and reduce intracellular oxidative stress levels. Klotho reduced $\mathrm{H}_{2} \mathrm{O}_{2}$-induced cellular damage and eventually maintained the osteogenic differentiation potential of hPDLSCs. Notably, Klotho promoted mitochondrial function and activated antioxidants by negatively regulating the PI3K/AKT/FoxO1 pathway. The findings suggest that Klotho protein enhanced the antioxidative ability of hPDLSCs and protected stem cell viability and stemness from $\mathrm{H}_{2} \mathrm{O}_{2}$-induced oxidative stress by restoring mitochondrial functions and the antioxidant system.
\end{abstract}

\section{Introduction}

Periodontitis is a chronic inflammatory disease that causes the destruction of tooth-supporting tissues and the progressive loss of periodontal attachment and alveolar bone $[1,2]$. Although periodontitis is the major cause of tooth loss in adults, treatments of periodontitis are far from satisfactory. Conventional infection control measures and regenerative approaches currently applied have shown limited efficacy on the restoration of periodontal supporting structures $[3,4]$. In recent years, stem cell-based bioengineered therapies have been investigated as therapeutic tools in regenerative medicine [5]. Mesenchymal stem cells (MSCs) are emerging as major sources for cell-based tissue engineer- ing due to their immunity privilege [6]. Human periodontal ligament stem cells (hPDLSCs) are MSCs from the periodontal ligament and the main candidate stem cells in periodontitis therapy. Being more accessible and possessing higher cell growth than human bone marrow stem cells (hBMMSCs) do, hPDLSCs have important homeostatic functions in vivo and display angiogenic, immunomodulatory, and multilineage differentiative capacity in vitro [7-9]. hPDLSCs have superior abilities to promote the formation of new bone, cementum, and periodontal ligament, achieving bone or periodontal tissue regeneration, as evidenced by accumulating studies [10-12]. With the osteoblastic differentiation ability, hPDLSCs are capable for repairing alveolar bone defect and periodontal intrabony defects [13, 14]. 
Exosomes derived from hPDLSCs also participate in mediating the immune balance and alleviating inflammatory microenvironment in periodontitis [15]. However, MSCs like hPDLSCs are placed in a harsh environment after isolation and transplantation, and the adverse microenvironment reduces their stemness and hinders their therapeutic effects [16].

Once MSCs are isolated from their original tissues or organs, they rapidly lose their vitality because of inappropriate ex vivo conditions. Additionally, long-term in vitro culture to increase cell number leads to a decreased colonyforming capacity $[17,18]$. Moreover, the transplantation of MSCs decreases their survival and proliferation rates because of low oxygen and nutrient supplies [19]. In such circumstances, MSCs will produce excessive reactive oxygen species (ROS), causing DNA damage and activating the apoptosis pathway. Consequently, oxidative stress impairs the selfrenewal, proliferation, and differentiation capacity of MSCs, leading to failed tissue regeneration [20]. High ROS levels can be generated by hydrogen peroxide $\left(\mathrm{H}_{2} \mathrm{O}_{2}\right)$, hydroxyl radicals, and superoxide anions in MSCs $[16,21]$.

Mitochondria are generally believed to play an important role in maintaining the normal ROS level [22]. Mitochondria are not only the main sites of ROS production but also important organelles in the antioxidant system [23, 24]. Under physiological conditions, MSCs will produce basal ROS to maintain cell proliferation and differentiation, and ROS are tightly regulated by the antioxidant system [25]. Under oxidative stress conditions, excessive ROS accumulates, increasing the antioxidative needs beyond the capacity of the antioxidant defence system [26]. Additionally, excessive ROS directly damage mitochondrial structure and function, concurrently leading to cell apoptosis and death $[22,27]$. Therefore, therapeutic antioxidative strategies that protect mitochondrial quality, improve antioxidative ability, and maintain the vitality and stemness of MSCs need to be developed.

Klotho is an antiageing protein that is predominantly expressed in the kidney and brain [28]. The human Klotho gene encodes three proteins: a full-length transmembrane form (mKL), a shed soluble form (sKL), and one secreted form produced by alternative splicing [29]. Klotho protein participates in the regulation of phosphate metabolism, energy metabolism, and stress resistance. Studies have found that secreted Klotho exerts antioxidative and antiinflammatory effects and that Klotho induces the expression of the manganese superoxide dismutase (MnSOD) protein, a mitochondrial antioxidant enzyme that detoxifies superoxides [30, 31]. Sahu et al. revealed that Klotho expression is highly related to the regeneration ability of muscle progenitor cells. The knockdown of the Klotho gene reduced mitochondrial function and cellular bioenergetics, coincident with declines in tissue regeneration $[32,33]$. Previous studies that reported Klotho protecting cardiac and renal cells against inflammatory responses and oxidative stress have provided new evidence for heart disease therapy and renal function recovery [30, 34, 35]. However, whether Klotho can exert a protective effect on hPDLSCs to maintain cell stemness under oxidative stress conditions is still unclear.
Therefore, the present study is aimed at investigating the effect of recombinant Klotho protein (same structure as secreted Klotho protein) on $\mathrm{H}_{2} \mathrm{O}_{2}$-induced oxidative stress in hPDLSCs. We demonstrated that Klotho protein enhanced antioxidative ability, reduced cellular damage, and protected osteogenic differentiation in hPDLSCs. Notably, we showed that Klotho maintained mitochondrial function and activated antioxidants, which were mediated by the AKT/FoxO1 pathway.

\section{Materials and Methods}

2.1. Cell Isolation and Culture. This research was approved by the Ethics Committee of Affiliated Stomatological Hospital of Sun Yat-sen University (ERC-[2016]-46). Human periodontal ligament tissues were obtained from healthy premolars from 12 donors (12-20 years old, 6 males and 6 females) with orthodontic demands. The middle section tissues of the root surfaces were scraped collected and enzymatically digested with $3 \mathrm{mg} / \mathrm{mL}$ collagenase type I and $4 \mathrm{mg} / \mathrm{mL}$ dispase for $1 \mathrm{~h}$. Subsequently, the tissues were cultured in a complete $\alpha$-minimum essential medium (Gibco, Grand Island, NY, USA) containing $10 \%(v / v)$ fetal bovine serum (Gibco), $100 \mathrm{U} / \mathrm{mL}$ penicillin, $100 \mu \mathrm{g} / \mathrm{mL}$ streptomycin (HyClone, Logan, UT, USA), and $5 \mathrm{mM}$ L-glutamine (Gibco) at $37^{\circ} \mathrm{C}$ in $5 \% \mathrm{CO}_{2}$. The hPDLSCs used in this study were a mixture of cells collected from 12 donors to decrease individual variation.

2.2. Cell Treatment and Cell Proliferation Assay. Cells in passages 3-5 were used in the experiments. hPDLSCs were seeded in 96-well plates at the density of $3 \times 10^{3}$ cells per well. $\mathrm{H}_{2} \mathrm{O}_{2}$ (Sigma-Aldrich, St. Louis, MO, USA) or Recombinant Klotho protein (PeproTech, Rocky Hill, NJ, USA) was added in the culture medium for $24 \mathrm{~h}$, tested by concentration gradient. Cell viability was assessed by the methylthiazolyldiphenyl-tetrazo-lium bromide (MTT) assay. $100 \mu \mathrm{L}$ of MTT $(5 \mathrm{mg} / \mathrm{mL})$ solution was added to each well and incubated for $4 \mathrm{~h}$ at $37^{\circ} \mathrm{C}$. Dimethyl sulfoxide was added and incubated for $10 \mathrm{~min}$ to dissolve the crystals. The absorbance was measured at a wavelength of $490 \mathrm{~nm}$ with an automatic microplate reader (BioTek, Winooski, VT, USA). To assess the effect of Klotho in reversing cell proliferation inhibition induced by $\mathrm{H}_{2} \mathrm{O}_{2}$, hPDLSCs were pretreated by various concentrations $(0-1000 \mathrm{ng} / \mathrm{mL})$ of Klotho for 24 hours, then treated by $100 \mu \mathrm{M} \mathrm{H}_{2} \mathrm{O}_{2}$ for 24 hours. After treatment, cell viability was tested by MTT assay as well.

2.3. Intracellular ROS Production. The intracellular ROS level was measured by the ROS assay kit (Beyotime Biotechnology, China). Briefly, the hPDLSCs were collected after different treatments, then incubated with DCFH-DA $(10 \mu \mathrm{M} / \mathrm{L})$ for $20 \mathrm{~min}$ at $37^{\circ} \mathrm{C}$, shaking slightly every $5 \mathrm{~min}$. The cells were washed with PBS, and the fluorescence was examined immediately under a fluorescence microscope (Carl Zeiss, Oberkochen, Germany) or flow cytometry (Beckman Coulter, Krefeld, Germany). Data were analyzed using CytExpert Software (Beckman Coulter). 
TABLE 1: Primer sequences used in quantitative real-time reverse transcription polymerase chain reaction.

\begin{tabular}{lc}
\hline Gene target & Sequence \\
\hline \multirow{2}{*}{ RUNX2 } & Forward: $5^{\prime}$-TGGTTACTGTCATGGCGGGTA-3' \\
& Reverse: $5^{\prime}$-TCTCAGATCGTTGAACCTTGCTA-3' \\
\hline \multirow{2}{*}{ BSP } & Forward: $5^{\prime}$-GAACCACTTCCCCACCTTTTG-3' \\
\hline \multirow{2}{*}{ GAPDH } & Reverse: $5^{\prime}$-ATTCTGACCATCATAGCCATCG-3' \\
\hline \multirow{2}{*}{ mtDNA } & Forward: 5' $5^{\prime}$-GGAGCGAGATCCCTCCAAAAT-3' \\
\hline \multirow{2}{*}{ Nuclear $\beta$-globin } & Reverse: $5^{\prime}$-GGCTGTTGTCATACTTCTCATGG-3' \\
\hline
\end{tabular}

2.4. Superoxide Dismutase (SOD) and Malondialdehyde (MDA) Activity Assay. The level of SOD and MDA were measured to assess the antioxidative stress effect of Klotho. The SOD activity of hPDLSCs was measured using a total SOD assay kit (Beyotime). The MDA level of hPDLSCs was tested by a lipid peroxidation MDA assay kit (Beyotime). Both were performed according to the manufacturer's instructions.

2.5. Cell Apoptosis Analysis. Cell apoptosis was quantitatively determined using the FITC Annexin V Apoptosis Detection Kit I (BD Biosciences, Franklin Lakes, NJ, USA) according to the manufacturer's protocol. hPDLSCs were collected and washed twice with cold PBS. Then, the cells were incubated in the solution containing FITC Annexin V, PI, and Binding Buffer for $15 \mathrm{~min}$ at $\mathrm{RT}\left(25^{\circ} \mathrm{C}\right)$ in the dark and finally analyzed by flow cytometry (Beckman Coulter).

2.6. Immunofluorescence. The hPDLSCs were seeded in a 12well plate under different treatments. Cells were fixed and permeabilized using $4 \%$ paraformaldehyde and $0.1 \%$ Triton $\mathrm{X}-100$. After blocking with $1 \%$ bovine serum albumin (BSA), the cells were incubated with cleaved Caspase-3 (cCaspase-3) (Cell Signaling, USA) as primary antibody at room temperature for $4 \mathrm{~h}$. Then, the cells were incubated with the secondary antibody conjugated to Alexa555 (Invitrogen, USA). Followed by incubation with Hoechst (Life Technologies, USA), the cells were imaged using fluorescence microscopy (Carl Zeiss).

2.7. Electron Microscopy. Mitochondrial structures were observed by transmission electron microscopy (TEM). hPDLSCs were fixed in $2.5 \%$ glutaraldehyde in $0.1 \mathrm{M}$ phosphate buffer for $2 \mathrm{~h}$ at $4^{\circ} \mathrm{C}$. Ultrathin sections were stained with uranyl acetate and lead citrate and photographed with a Tecnai $G^{2}$ Spirit Twin TEM (FEI, USA). Sections were scanned randomly at 10 different spots per sample at $\times 18500$ magnification. The numbers and sizes of mitochondria were measured using imaging software (ImageJ).
2.8. Mitochondrial Membrane Potential Detection $(\Delta \Psi m)$. The $\Delta \Psi \mathrm{m}$ of hPDLSCs was measured using a mitochondrial membrane potential assay kit with JC-1 probe (Beyotime). When live cells are incubated with JC-1, the mitochondria are driven by the $\Delta \Psi \mathrm{m}$ and JC- 1 is rapidly taken up, raising the JC- 1 concentration and the formation of aggregates (Jaggregates) within the mitochondria, which provoke red fluorescence. However, JC-1 does not accumulate in depolarized mitochondria with low $\Delta \Psi \mathrm{m}$ and remains in the cytoplasm as monomers, which emit green fluorescence. hPDLSCs were incubated in JC-1 working solution at $37^{\circ} \mathrm{C}$ for $20 \mathrm{~min}$, then were washed twice with wash buffer and measured by flow cytometric analysis (Beckman Coulter) or using a fluorescent microscope (Carl Zeiss).

2.9. Alizarin Red Staining. hPDLSCs were seeded into 12 well plates at a density of $4 \times 10^{4}$ cells/well. hPDLSCs were treated the same as above. After reaching $80 \%$ confluence, cells were cultured in an osteogenic induction medium (phenol red-free $\alpha$-MEM supplemented with $10 \% \mathrm{FBS}, 10 \mathrm{mmol} / \mathrm{L} \beta$-glycerophosphate, $10 \mathrm{nmol} / \mathrm{L}$ dexamethasone, and $50 \mu \mathrm{g} / \mathrm{mL}$ ascorbic acid) for osteogenic differentiation. The medium was replaced every other day. 14 days later, cells were stained by the Alizarin red ( $\mathrm{pH} 4.2$, Alfa Assar). Calcium nodules were observed via a microscope and imaged with camera. For quantitative analysis, stained nodules were dissolved with $10 \%(w / v)$ cetylpyridinium chloride (Sigma-Aldrich) and detected by an automatic microplate reader at $562 \mathrm{~nm}$.

2.10. Alkaline Phosphatase (ALP) Activity. After 7 days of osteogenic induction, hPDLSCs were fixed and stained according to the manufacturer's instructions of the BCIP/NBT alkaline phosphatase colour development kit (Beyotime) and the images were captured with a camera. ALP activity of hPDLSCs was measured following the manufacturer's instructions using the Alkaline Phosphatase Assay kit (Beyotime). Absorbance was evaluated spectrophotometrically at $405 \mathrm{~nm}$. 


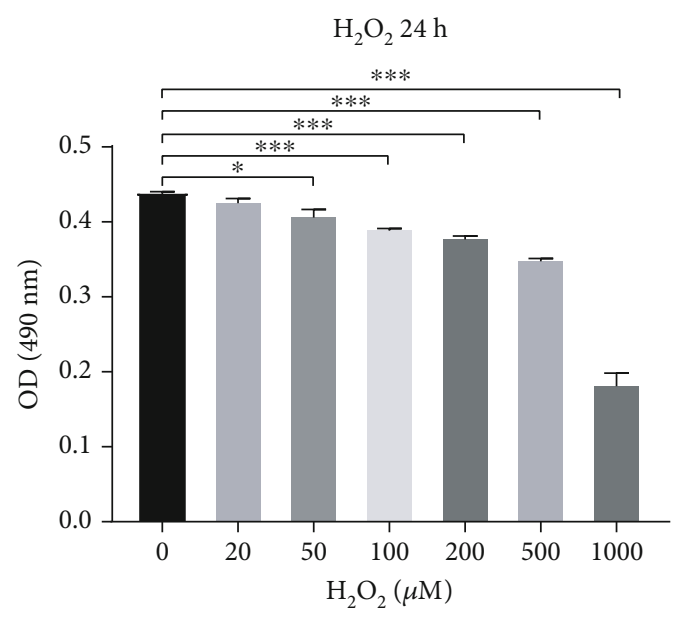

(a)

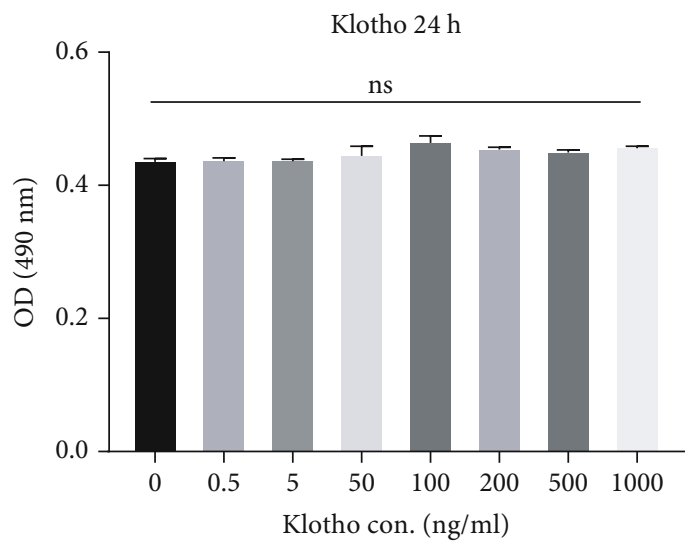

(b)

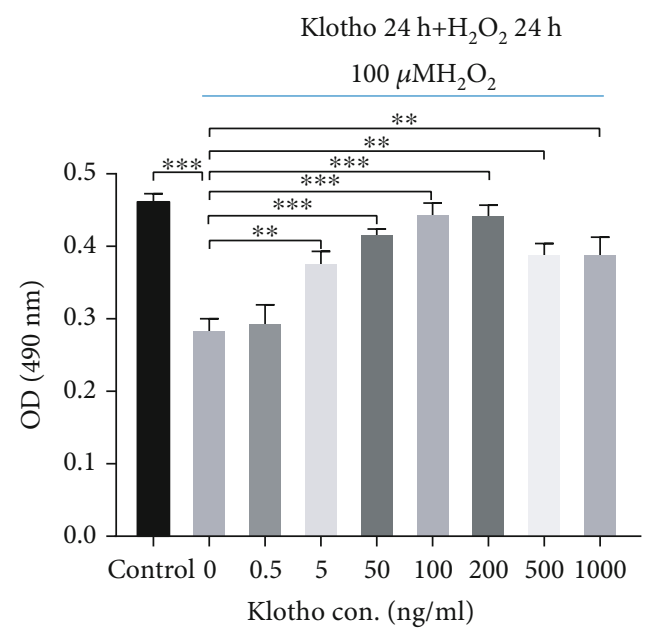

(c)

Figure 1: Effects of $\mathrm{H}_{2} \mathrm{O}_{2}$ and Klotho on hPDLSC proliferation. (a) Effect of $\mathrm{H}_{2} \mathrm{O}_{2}$ on the proliferation of hPDLSCs. (b) Effect of Klotho on the proliferation of hPDLSCs under normal culture conditions. (c) Effect of Klotho on the proliferation of hPDLSCs under oxidative stress conditions. Control: without Klotho or $\mathrm{H}_{2} \mathrm{O}_{2}$ treatment. ${ }^{*} P<0.05,{ }^{* *} P<0.01$, and ${ }^{* * *} P<0.001$; ns: no statistical significance.

\subsection{Quantitative Real-Time Reverse Transcription} Polymerase Chain Reaction ( $q R T-P C R$ ). Total RNA samples were isolated from cells by using the Ultrapure RNA kit (CWBIO, China). mRNA was reverse transcribed into cDNA by a Reverse Transcriptase M-MLV Kit (TaKaRa, Japan) according to the manufacturer's instructions. RT-PCR was performed on the LightCycler ${ }^{\circledR} 480$ Real-Time PCR System with LightCycler ${ }^{\circledR} 480$ SYBR-Green I Master (Roche Diagnostics, Swiss). The amplification conditions were set as follows: $95^{\circ} \mathrm{C}$ for $10 \mathrm{~min}, 40$ cycles of denaturation at $95^{\circ} \mathrm{C}$ for $15 \mathrm{~s}$, annealing at $60^{\circ} \mathrm{C}$ for $20 \mathrm{~s}$, and final extension at $72^{\circ} \mathrm{C}$ for $20 \mathrm{~s}$. GAPDH was used as the internal reference gene. Gene expression was calculated using the $2^{-\Delta \Delta \mathrm{Ct}}$ method. Primer sequences are listed in Table 1.

2.12. Western Blot Analysis. hPDLSCs were lysed in RIPA buffer at $4^{\circ} \mathrm{C}$ for $30 \mathrm{~min}$. Protein concentrations were measured by a Pierce BCA Protein Assay Kit (Thermo Scientific; Pierce, Germany). The sample protein was separated on $10 \%$ sodium dodecyl sulfate polyacrylamide gels and electrotrans- ferred to nitrocellulose membranes (Millipore, Germany). Subsequently, membranes were incubated in 5\% nonfat milk for $1 \mathrm{~h}$ at room temperature and then incubated overnight with the primary antibodies at $4^{\circ} \mathrm{C}$. Finally, the membranes were washed with TBST buffer and incubated with HRPlinked antibody for $1 \mathrm{~h}$ at room temperature, followed by detection using the GeneGnome XRQ chemiluminescence imaging system. Primary antibodies against the following were used in this study: RUNX2 (ABclonal, China), BSP (Boster, China), AKT (Cell Signaling), phospho-AKT (PAKT) (Cell Signaling), FoxO1 (Cell Signaling), phosphoFoxO1 (P-FoxO1) (Cell Signaling), Catalase (Cell Signaling), MnSOD (Cell Signaling), and $\beta$-actin (Sigma-Aldrich). Relative density of protein band was analyzed by ImageJ software and normalized to $\beta$-actin.

2.13. Mitochondrial DNA ( $m t D N A)$ Copy Number. Nuclear and mitochondrial DNA of hPDLSCs was isolated according to a HiPure Tissue DNA Mini Kit using the manufacturer's protocol (Magen, China). The relative mtDNA copy 


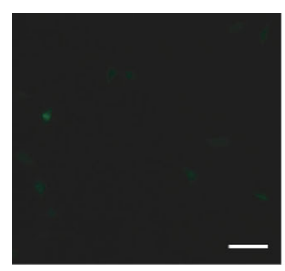

Control

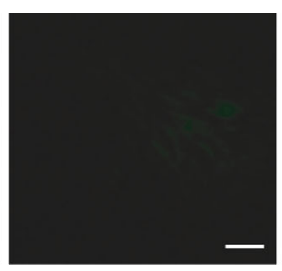

Klotho

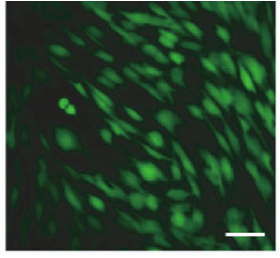

$\mathrm{H}_{2} \mathrm{O}_{2}$

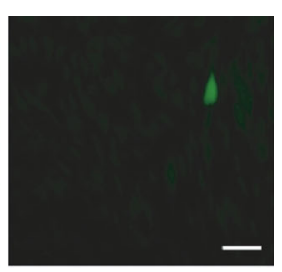

Klotho $+\mathrm{H}_{2} \mathrm{O}_{2}$

(a)
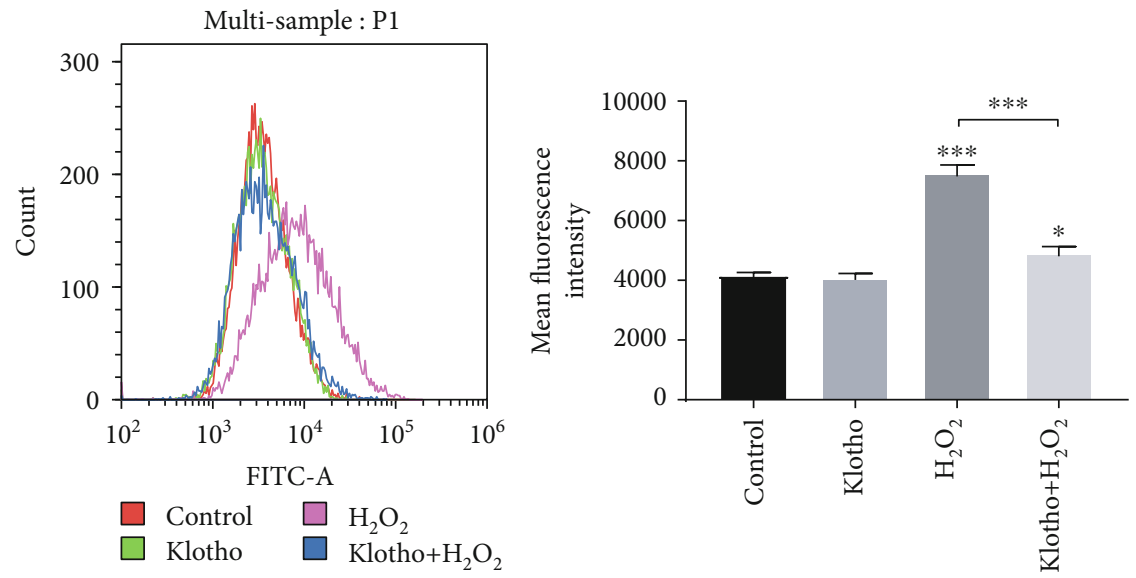

(b)

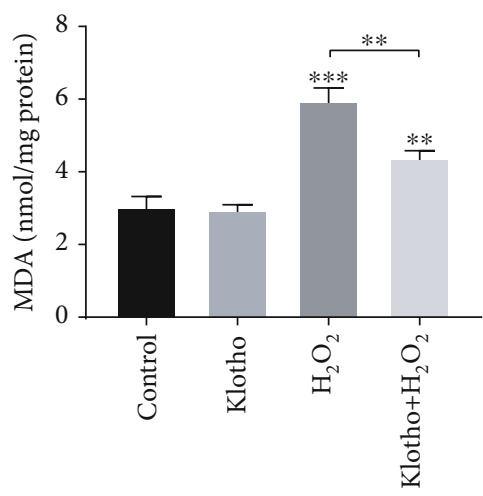

(c)

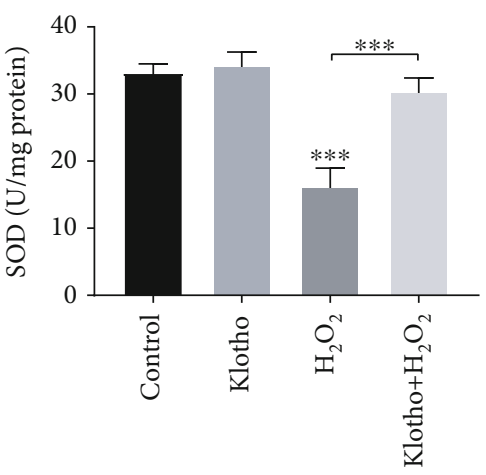

(d)

FIGURE 2: Klotho attenuated $\mathrm{H}_{2} \mathrm{O}_{2}$-induced intracellular oxidative stress status. SOD activity, MDA formation, and ROS production were assessed. ( $a, b)$ The level of ROS in hPDLSCs was measured by DCFH-DA through a fluorescence microscope (a) and flow cytometry (b); (c) the MDA level and (d) SOD level in hPDLSCs. Scale bars: $50 \mu \mathrm{m}$. Magnification: $200 \mathrm{x} .{ }^{*} P<0.05,{ }^{* *} P<0.01$, and ${ }^{* * *} P<0.001$ versus control group.

number was quantified by real-time polymerase chain reaction (PCR) and normalized by simultaneous measurement of the nuclear DNA according to the method described in other studies [36]. The primer sequences L394 and H475 were used for measuring the mtDNA content, and the primers HBG1F and HBG1R were used for amplification of the single-copy nuclear $\beta$-globin gene (seen in Table 1). Nuclear $\beta$-globin was used as the internal control.

2.14. Mitochondrial Bioenergetic Assessment. The cellular oxygen consumption rate (OCR) was assessed in real-time using a Seahorse XFe96 Extracellular Flux Analyzer (Billerica, MA, USA). Before running the procedure, the cell medium was changed to a running medium (DMEM supplemented with $5.5 \mathrm{mM}$ glucose, $1 \mathrm{mM}$ sodium pyruvate, $4 \mathrm{mM}$ L-glutamine, $\mathrm{pH} 7.4$ ) and incubated at $37^{\circ} \mathrm{C}$ in a non- $\mathrm{CO}_{2}$ incubator for $1 \mathrm{~h}$. The basal OCR was measured by averaging the OCR values before treating the cells with oligomycin. Total reserve capacity was calculated by the differences of OCR between treatment with FCCP and 2DG and basal values.

2.15. Statistical Analysis. All experiments were performed three times separately, and all data were expressed as mean \pm S.D. Data were analyzed by using one-way ANOVA and SPSS20.0 software package (SPSS Inc., Chicago, IL, USA). $P<0.05$ indicated significance. 

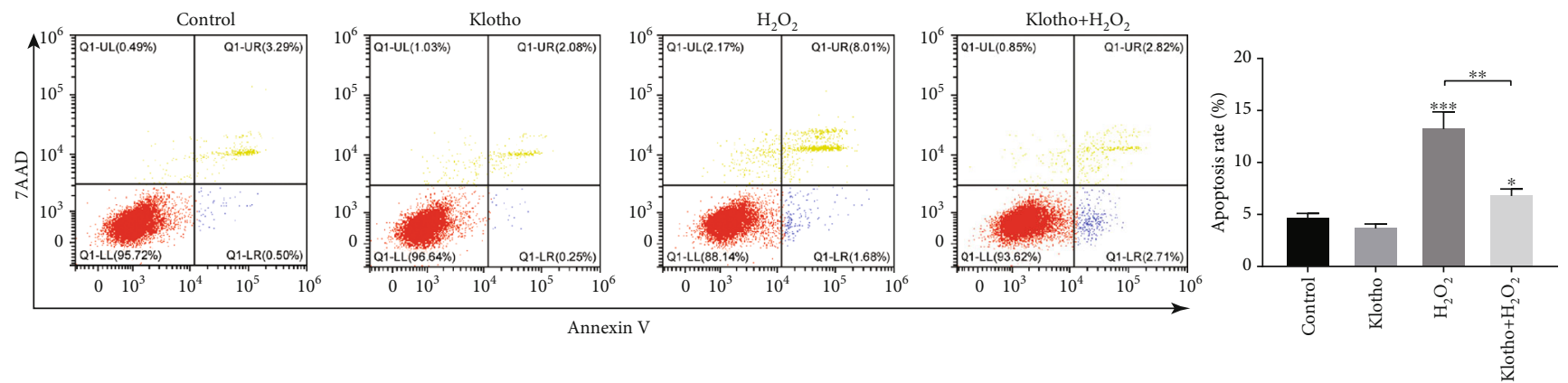

(a)
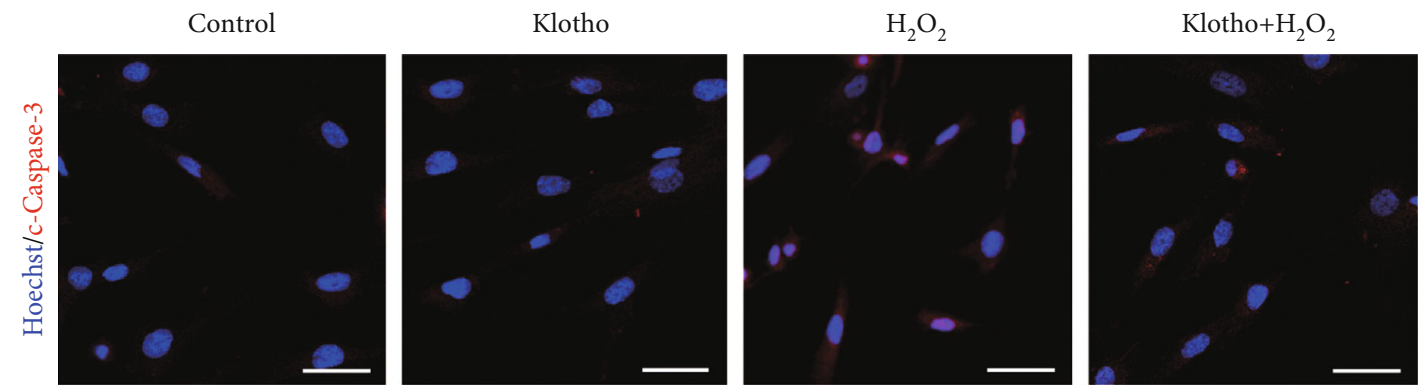

(b)

FIGURE 3: Klotho administration ameliorated $\mathrm{H}_{2} \mathrm{O}_{2}$-induced apoptosis in hPDLSCs. (a) Flow cytometric analysis of groups with different treatments and the percentage of Annexin V/7-AAD positive cells. (b) The expression of c-Caspase-3 in hPDLSCs with different treatments was detected by immunofluorescence staining. Scale bars: $50 \mu \mathrm{m}$. Magnification: $400 \mathrm{x} .{ }^{*} P<0.05,{ }^{* *} P<0.01$, and ${ }^{* * *} P<0.001$.

\section{Results}

3.1. Recombinant Klotho Protein Protected hPDLSC Proliferation from $\mathrm{H}_{2} \mathrm{O}_{2}$-Induced Cytotoxicity. The isolated cells were plastic-adherent and exhibited colony-forming ability. Flow cytometry analysis showed that the cells expressed CD90 and CD105 (more than 99\%) and did not express CD34, CD45, and HLA-DR surface molecules (less than 1\%). Moreover, the cells possessed the ability to differentiate into osteoblasts and adipocytes. These results suggested that the cells we isolated from human periodontal ligament tissue were MSCs (Figure S1). To determine the appropriate concentrations of $\mathrm{H}_{2} \mathrm{O}_{2}$ and Klotho, we used the MTT assay to assess the viability of hPDLSCs treated with different concentrations of $\mathrm{H}_{2} \mathrm{O}_{2}$ and Klotho, respectively. As shown in Figure 1(a), the proliferative activity of hPDLSCs was significantly decreased following treatment with concentrations of $\mathrm{H}_{2} \mathrm{O}_{2}$ at $100 \mu \mathrm{M}$ and above and was sharply reduced when the cells were exposed to 500 and $1000 \mu \mathrm{M} \mathrm{H}_{2} \mathrm{O}_{2}$. Therefore, $100 \mu \mathrm{M}$ was chosen as the moderate $\mathrm{H}_{2} \mathrm{O}_{2}$ concentration to establish the oxidative stress model. The viability of hPDLSCs treated with different concentrations of Klotho was not significantly changed, suggesting that Klotho has no cytotoxicity on hPDLSCs (Figure 1(b)). To investigate whether Klotho ameliorates $\mathrm{H}_{2} \mathrm{O}_{2}$-induced cytotoxicity, we assessed the cell viability of hPDLSCs pretreated with different concentrations of Klotho before $\mathrm{H}_{2} \mathrm{O}_{2}$. The results showed that Klotho ameliorated $\mathrm{H}_{2} \mathrm{O}_{2}$-induced cytotoxicity in hPDLSCs, and the effect peaked at a concentration of $100 \mathrm{ng} / \mathrm{mL}$ (Figure 1(c)). Based on these results, $100 \mathrm{ng} / \mathrm{mL}$ Klotho was chosen for the subsequent experiments.

3.2. Recombinant Klotho Protein Attenuated Intracellular Oxidative Stress Status in hPDLSCs. To verify the effect of Klotho on $\mathrm{H}_{2} \mathrm{O}_{2}$-induced intracellular oxidative stress status, we assessed ROS production, MDA formation, and SOD activity in hPDLSCs. ROS and MDA levels were increased, and SOD activity was impaired by $\mathrm{H}_{2} \mathrm{O}_{2}$ treatment compared with the control condition. However, ROS production and MDA formation were reduced by $100 \mathrm{ng} / \mathrm{mL}$ Klotho pretreatment (Figures 2(a)-2(c)), and SOD activity was also restored with the addition of Klotho (Figure $2(\mathrm{~d})$ ), indicating that Klotho attenuates $\mathrm{H}_{2} \mathrm{O}_{2}$-induced oxidative stress and exerts an antioxidative effect on hPDLSCs under mimicking oxidative stress status.

3.3. Recombinant Klotho Protein Reduced $\mathrm{H}_{2} \mathrm{O}_{2}$-Stimulated Oxidative Cellular Damages in hPDLSCs. To investigate whether Klotho decreases cell apoptosis, flow cytometric analysis was performed by Annexin V staining. The results showed that Annexin V/7-AAD-positive hPDLSCs were increased in the presence of $\mathrm{H}_{2} \mathrm{O}_{2}$ and were significantly reduced by Klotho pretreatment (Figure 3(a)). The expression of c-Caspase-3, a proapoptotic marker, was detected by immunofluorescence staining. We found that $c$-Caspase- 3 was activated in hPDLSCs in the presence of $\mathrm{H}_{2} \mathrm{O}_{2}$ and that Klotho pretreatment reduced the expression of c-Caspase- 3 (Figure 3(b)). 

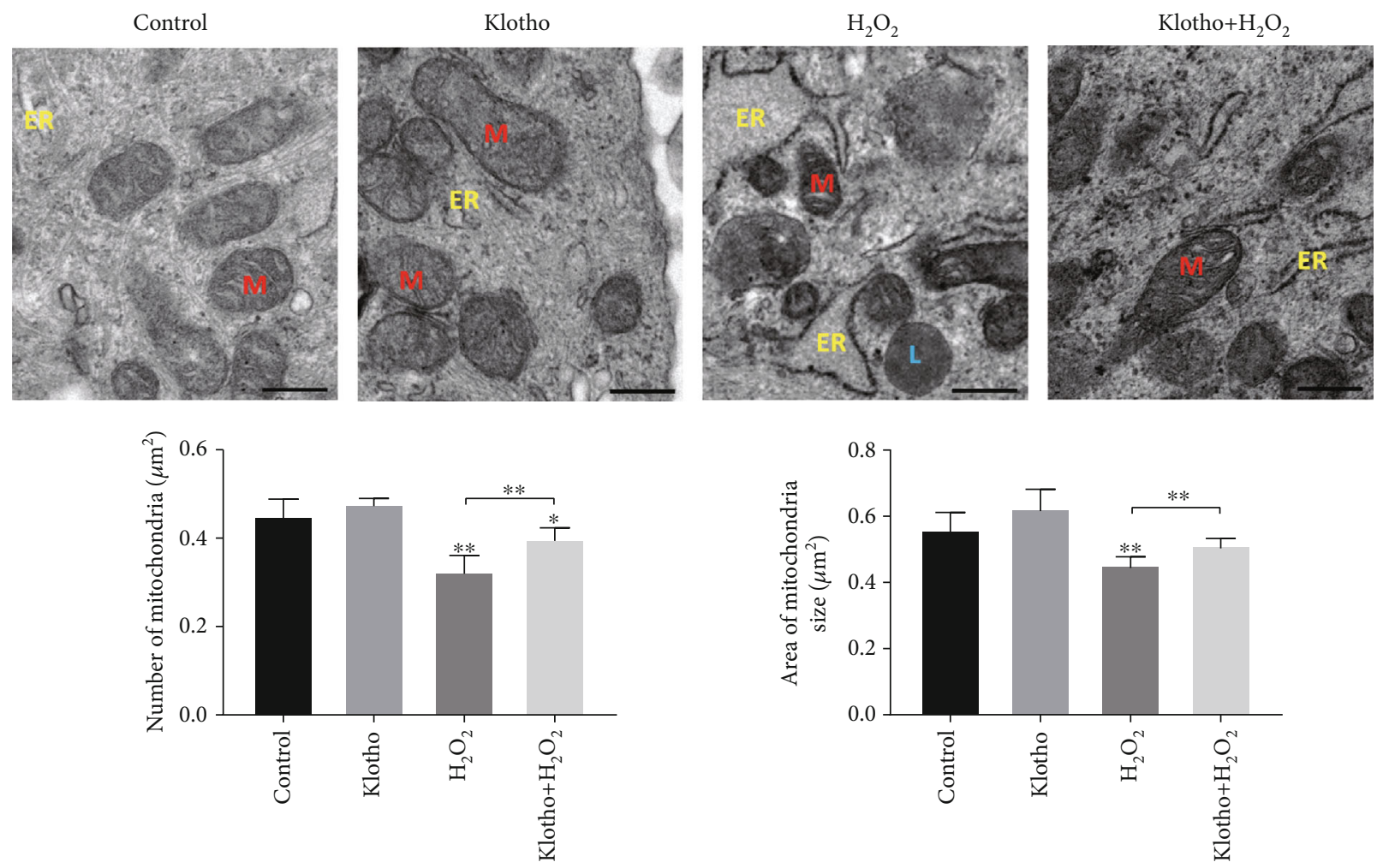

(a)
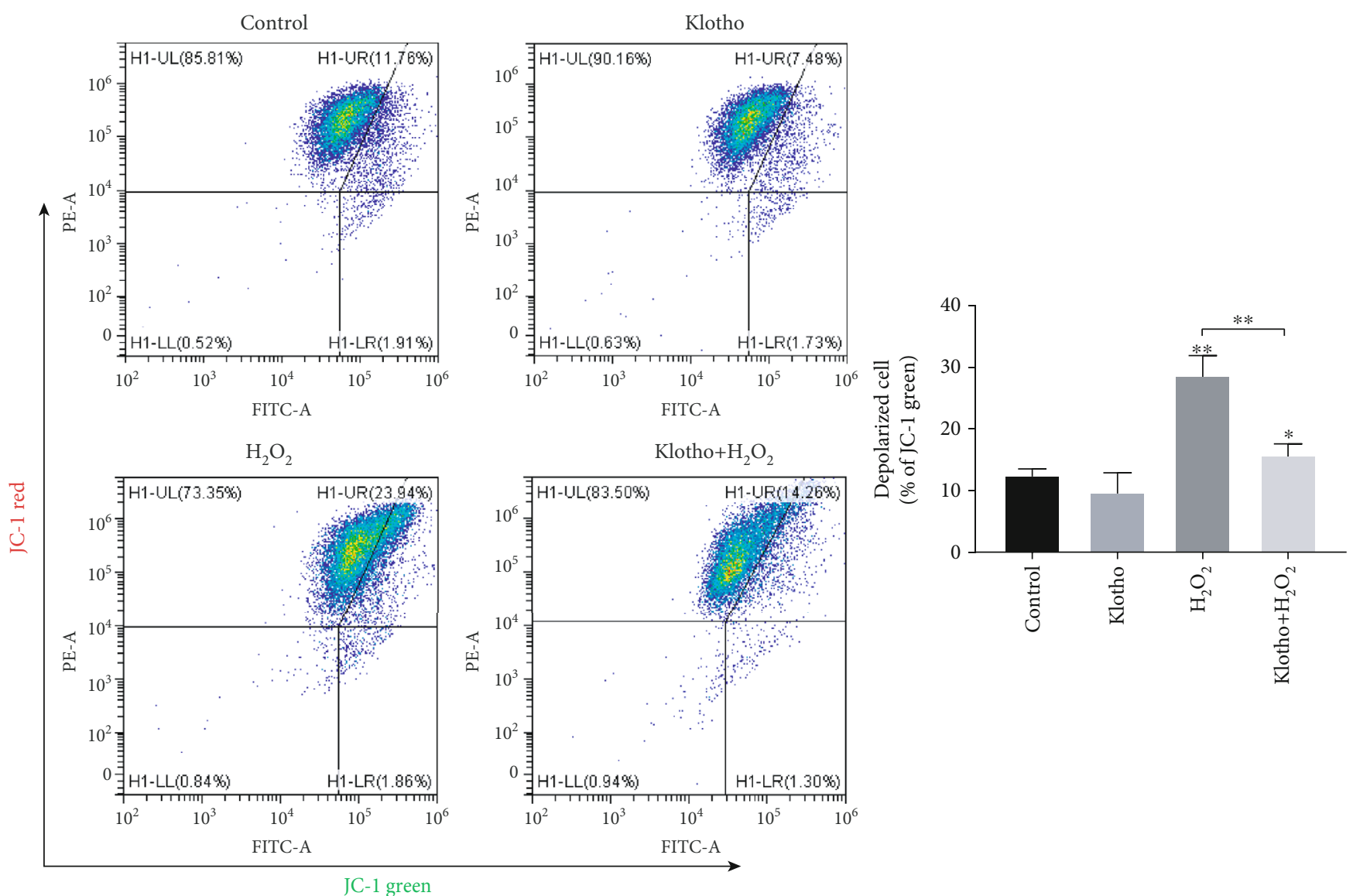

JC-1 green

(b)

Figure 4: Continued. 

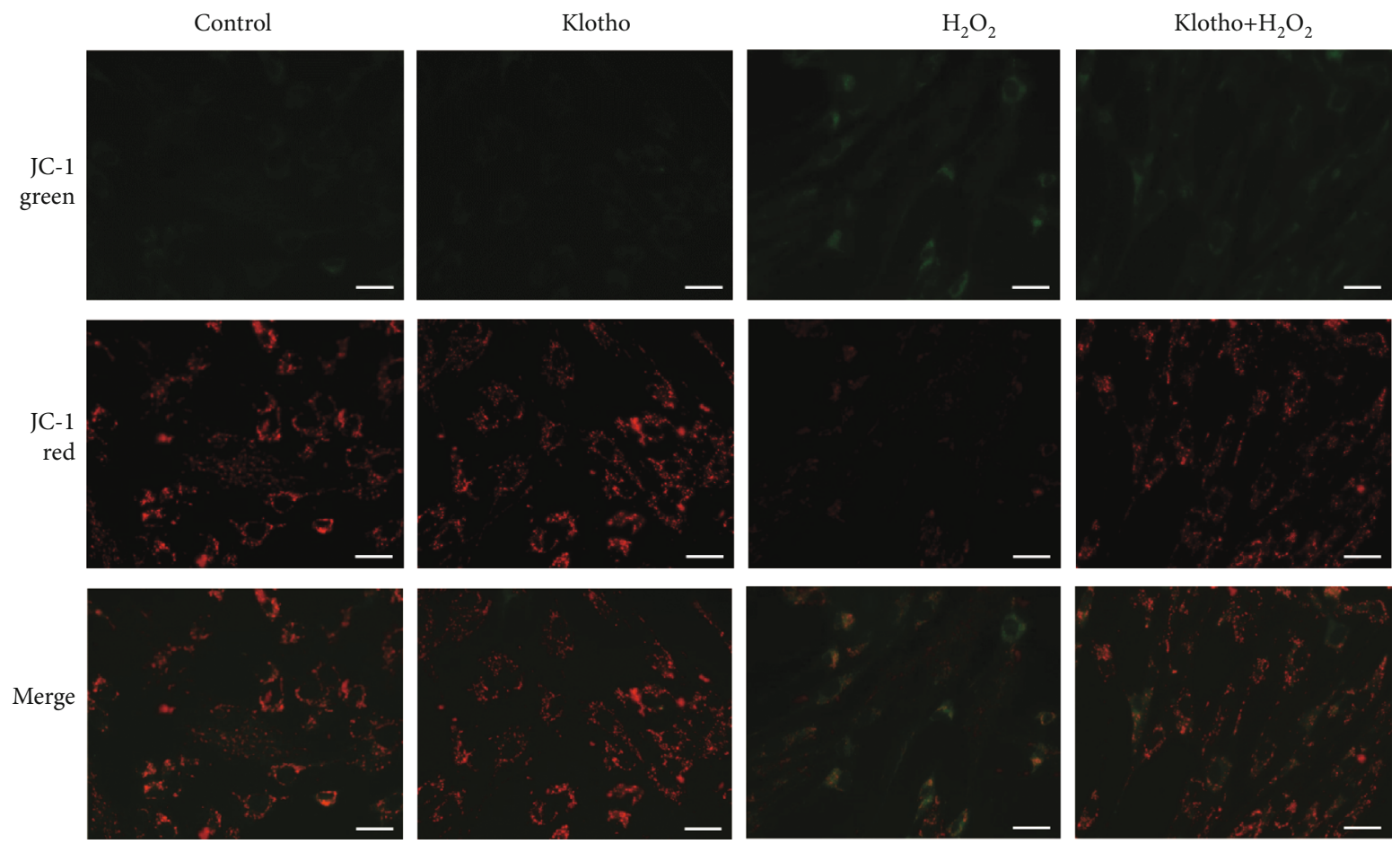

(c)

Figure 4: Klotho administration ameliorated $\mathrm{H}_{2} \mathrm{O}_{2}$-induced mitochondrial destruction in hPDLSCs. (a) Representative transmission electron microscopy images of mitochondria and their quantification in each group. (b) Flow cytometry and (c) fluorescent microscopy images of the mitochondrial membrane potential in PDLSCs with different treatments. JC-1 aggregates show red fluorescence, indicating high mitochondrial membrane potential, and JC-1 monomers show green fluorescence. M: mitochondria; L: lipid droplet; ER: endoplasmic reticulum. Scale bars: $0.5 \mu \mathrm{m}$ (a) or $30 \mu \mathrm{m}$ (c). Magnification: $18500 \mathrm{x}$ (a) or $200 \mathrm{x}$ (c). ${ }^{*} P<0.05,{ }^{* *} P<0.01$, and ${ }^{* * *} P<0.001$.

To identify the effect of Klotho on mitochondrial structure, we studied morphological changes in the mitochondria of hPDLSCs by electron microscopy. Ultrastructural analysis revealed that $\mathrm{H}_{2} \mathrm{O}_{2}$ treatment caused a reduction in the number, and size of mitochondria, and Klotho pretreatment overcame those changes (Figure 4(a)). The collapse of mitochondrial membrane potential is often observed during early apoptosis [37]. Next, the $\Delta \Psi \mathrm{m}$ of hPDLSCs administered different treatments was measured (Figure 4(b)). The results showed that the number of depolarized cells with low $\Delta \Psi \mathrm{m}$ was increased in the $\mathrm{H}_{2} \mathrm{O}_{2}$ group, but Klotho administration ameliorated this impairment, indicating that Klotho may exert an effect on mitochondrial structure maintenance.

3.4. Recombinant Klotho Protein Alleviated the Suppression of $\mathrm{H}_{2} \mathrm{O}_{2}$-Induced Osteogenic Differentiation in hPDLSCs. To verify the effect of Klotho on the osteogenic differentiation of hPDLSCs in $\mathrm{H}_{2} \mathrm{O}_{2}$-induced oxidative stress, we assessed extracellular matrix (ECM) mineralization, ALP activity, and the mRNA and protein expression of osteogenicrelated markers. The formation of mineral nodules and ALP activity was inhibited under $\mathrm{H}_{2} \mathrm{O}_{2}$ stimulation and was reversed by Klotho pretreatment (Figure 5(a)). RUNX2 and BSP play crucial roles in osteogenic differentiation. RT-PCR and Western blot analysis showed that the expression of RUNX2 and BSP was downregulated remarkably in the $\mathrm{H}_{2} \mathrm{O}_{2}$ group, and Klotho pretreatment elevated osteogenic gene expression (Figures 5(b) and 5(c)). These results demonstrated that Klotho protected osteogenic potential, which represents the stemness of hPDLSCs, from $\mathrm{H}_{2} \mathrm{O}_{2}$-induced oxidative stress.

3.5. Recombinant Klotho Protein Contributed to the Regulation of Mitochondrial Dysfunction in hPDLSCs. We have demonstrated that Klotho exerts protective effects on hPDLSC mitochondrial structure. Next, we evaluated whether Klotho protein has a direct influence on hPDLSC mitochondrial function in an oxidative environment. qRTPCR results showed that mitochondrial DNA (mtDNA) copy number, a marker representing mitochondrial viability, was significantly reduced in the $100 \mu \mathrm{M} \mathrm{H}_{2} \mathrm{O}_{2}$ group compared with the control group and could be ameliorated by Klotho pretreatment (Figure 6(a)). The OCR of cells was assessed and shown in Figures 6(b)-6(e). The OCR of Klothopretreated cells was higher than that of cells treated with $\mathrm{H}_{2} \mathrm{O}_{2}$ alone. Klotho-pretreated hPDLSCs showed significantly higher basal respiration, maximal respiration, and spare respiratory capacity. Overall, these data supported the hypothesis that Klotho could protect hPDLSC mitochondrial bioenergetics from $\mathrm{H}_{2} \mathrm{O}_{2}$-induced oxidative stress.

3.6. Recombinant Klotho Protein Enhanced the Antioxidant System by Regulating the PI3K/AKT/FoxO1 Pathway. The $\mathrm{PI} 3 \mathrm{~K} / \mathrm{AKT}$ signalling pathway plays a crucial role in stem cell self-renewal and differentiation, and its downstream target 


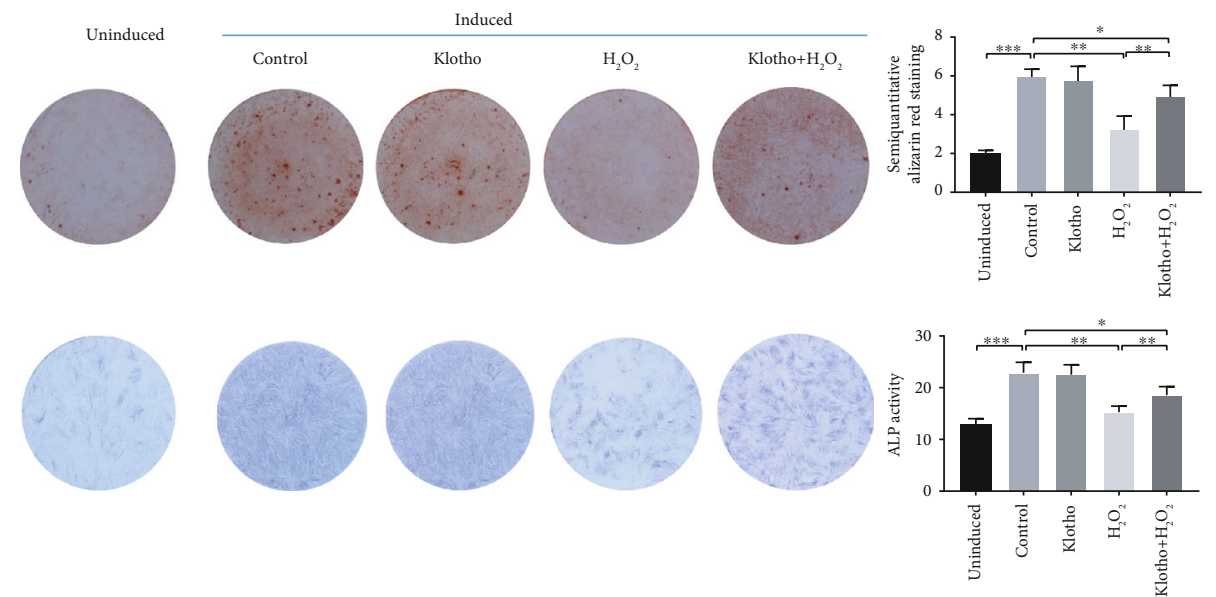

(a)

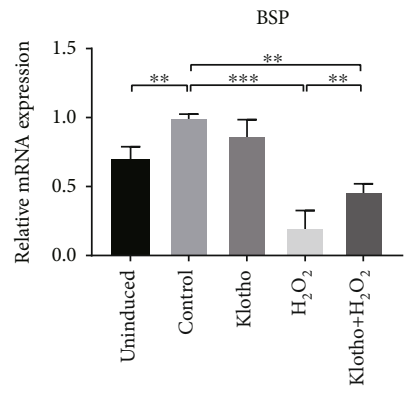

(b)

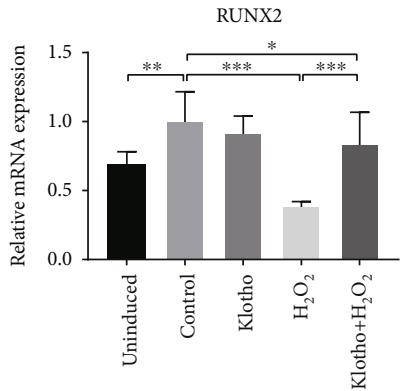

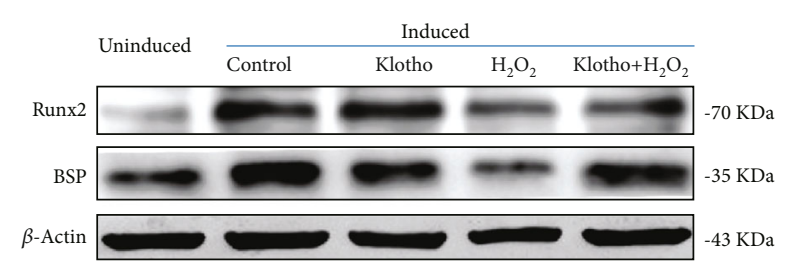

(c)

FIgURe 5: The effect of Klotho on the osteogenic differentiation of hPDLSCs stimulated by $\mathrm{H}_{2} \mathrm{O}_{2}$. (a) Alizarin red staining and ALP activity assay. (b) qRT-PCR analysis of RUNX2 and BSP mRNA expression. (c) Western blot analysis of RUNX2 and BSP gene expression. ${ }^{*} P<0.05$, ${ }^{* *} P<0.01$, and ${ }^{* * *} P<0.001$.

FoxO1 is tightly related to antioxidant genes, such as MnSOD and Catalase $[38,39]$. Therefore, we explored the effect of Klotho on PI3K/AKT/FoxO1 pathway regulation. Western blot analysis showed that $\mathrm{H}_{2} \mathrm{O}_{2}$ activated the AKT/FoxO1 pathway in hPDLSCs, promoting the phosphorylation of AKT and FoxO1. Additionally, $\mathrm{H}_{2} \mathrm{O}_{2}$ reduced total FoxO1 expression, leading to the reduced expression of Catalase and MnSOD. Notably, the $\mathrm{H}_{2} \mathrm{O}_{2}$-induced decrease in enzymatic antioxidants was reversed by Klotho pretreatment, which is consistent with the elevated FoxO1 expression (Figures 7(a) and 7(b)). Taken together, the results show that Klotho negatively regulates the PI3K/AKT/FoxO1 signalling pathway and subsequently enhances FoxO1-mediated antioxidant expression.

\section{Discussion}

Since the concept of tissue engineering was proposed two decades ago [40], MSCs have been extensively studied because of their significant potential in the field of regenerative medicine [41]. However, so far, MSCs are much farther from reaching clinical utility in regenerative medicine than they are in immunomodulation [42]. One of the important reasons is the effect of oxidative stress on MSC ex vivo expansion and in vivo engraftment, leading to the loss of stemness and low survival rate in transplant sites, eventually causing failed tissue regeneration [16, 21]. Excessive ROS in MSCs is the typical sign for oxidative stress in the injured microenvironment.

In our study, we aimed to find a solution to improve hPDLSC bioactivity under oxidative stress. Accumulating evidence suggests that excessive oxidative stress and diminished antioxidant defences could contribute to age-related tissue damage and diseases [43]. The antiageing protein Klotho exerts antioxidative effects in cells [31]. In the present study, we investigated whether exogenous treatment with Klotho protein could protect against $\mathrm{H}_{2} \mathrm{O}_{2}$-induced oxidative injury in hPDLSCs.

$\mathrm{H}_{2} \mathrm{O}_{2}$ has been extensively used on in vitro models to induce oxidative stress [44]. ROS overproduction and failed antioxidant defence lead to redox imbalance and increased oxidative stress [45]. $\mathrm{H}_{2} \mathrm{O}_{2}$ causes cell oxidative damage mainly by causing excessive ROS and attacking the antioxidant system [46]. We found that $100 \mathrm{ng} / \mathrm{mL}$ Klotho protein showed the greatest protection against oxidative stress. The excess accumulation of ROS damages polyunsaturated fatty acids and leads to higher levels of MDA, which is a major end product of membrane lipid peroxidation [47]. Lipid peroxidation may cause cell membrane damage, resulting in a decreased membrane permeability and fluidity and eventual cell death [48]. Exogenous proteins exert antioxidative effects by promoting the expression of antioxidant enzymes such as SOD [49]. By detecting the level of oxidative stress biomarkers [50], we found that Klotho alleviated oxidative stress 


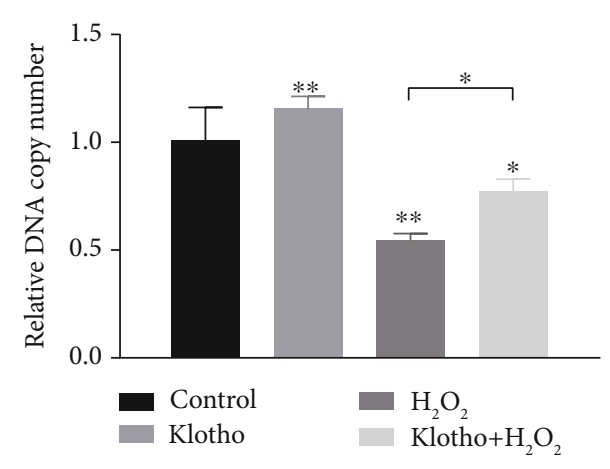

(a)

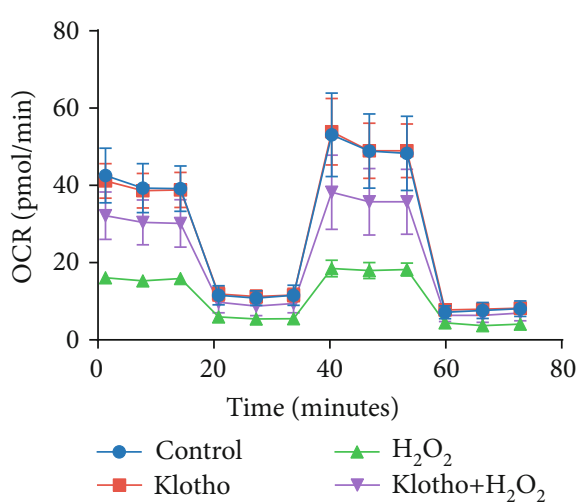

(b)

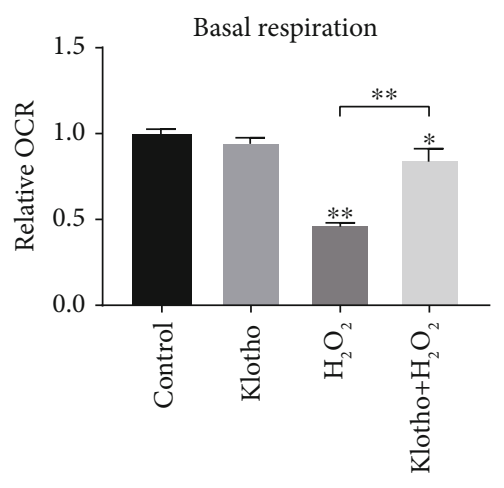

(c)

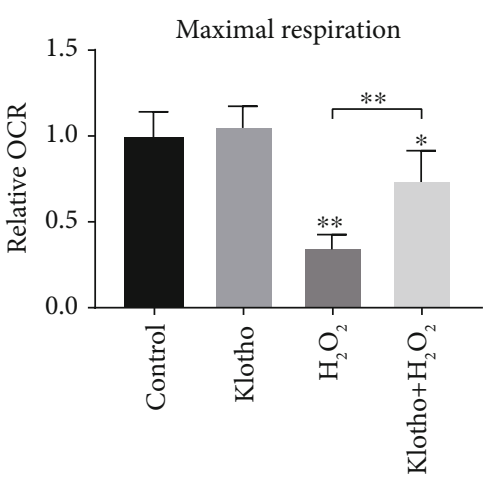

(d)

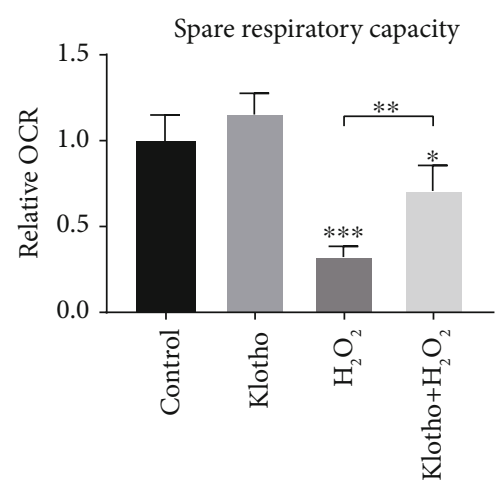

(e)

FIGURE 6: The effect of Klotho on the mitochondrial function of hPDLSCs stimulated by $\mathrm{H}_{2} \mathrm{O}_{2}$. (a) mtDNA copy number analysis by qRTPCR. (b) Seahorse analysis of hPDLSCs. The areas under the curve for respiration (OCR) are shown, and (c-e) the basal, maximal, and spare respiration capacity were calculated from the OCR. ${ }^{*} P<0.05,{ }^{* *} P<0.01$, and ${ }^{* * *} P<0.001$.

in hPDLSCs by reducing ROS production and lipid oxidative damage, while the activities of antioxidant enzymes such as SOD were maintained (Figure 2).

The TEM observations showed that the hPDLSCs pretreated with Klotho maintained mitochondrial ultrastructure (Figure 4(a)). Klotho reduced vacuolated mitochondria and compromised cristae structure under $\mathrm{H}_{2} \mathrm{O}_{2}$-induced oxidative stress. The mitochondrial membrane depolarization induced by $\mathrm{H}_{2} \mathrm{O}_{2}$ treatment was also recovered by Klotho treatment (Figures 4(b) and 4(c)). A reduction in mitochondrial membrane potential induces the opening of the mitochondrial permeability transition pore and the release of proapoptosis factors, such as cytochrome $c$, into the cytosol [51]. These previous results explained the findings in our study that cellular apoptosis paralleled mitochondrial damage, and Klotho reduced the $\mathrm{H}_{2} \mathrm{O}_{2}$-induced cell apoptosis rate and caspase 3 expression level (Figure 3). $\mathrm{H}_{2} \mathrm{O}_{2}$ exposure can induce mitochondrial fission and apoptosis by converting cytochrome $c$ into an oxidant-generating peroxidase, which eventually impairs cellular dynamics [52]. The present results show that the Klotho protein attenuated the hPDLSC cellular damage and cell apoptosis induced by oxidative stress by protecting mitochondrial structure. Other studies showed a similar function of Klotho, reporting that it reduces cell apoptosis by decreasing mitochondrial ROS production $[53,54]$.
hPDLSCs possess predominant osteogenic differentiation potentials and are supposed to be ideal cell sources for periodontal regeneration [55]. However, $\mathrm{H}_{2} \mathrm{O}_{2}$ impairs osteogenic capacity by suppressing ALP activity, mineralization, and osteogenic gene levels, including the levels of RUNX2, BSP, and OCN [56]. A previous report showed that the addition of antioxidants reduces ROS generation and eliminates oxidative stress, strikingly elevating the expression of RUNX2, ALP, and OPG [57]. We found that Klotho increased the osteogenic differentiation of PDLSCs under oxidative stress, promoting mineralized nodule deposition, ALP activity, and RUNX2 and BSP expression (Figure 5). From the data, we concluded that Klotho protected the osteogenic differentiation of hPDLSCs against oxidative stress, showing the potential to solve problems in periodontal regeneration.

However, it is not known how Klotho exerts these effects on hPDLSC osteogenesis. Studies have found that increased mtDNA copy number and enhanced mitochondrial respiration were observed in differentiated MSCs, indicating that mitochondria may participate in the osteogenic differentiation of MSCs [58]. We further investigated mitochondrial function in different groups, and the results demonstrate that the antioxidative effect promoted by Klotho coincides with the regulation of mitochondrial bioenergetics. Mitochondrial function, as assessed by mtDNA damage and OCR, showed 


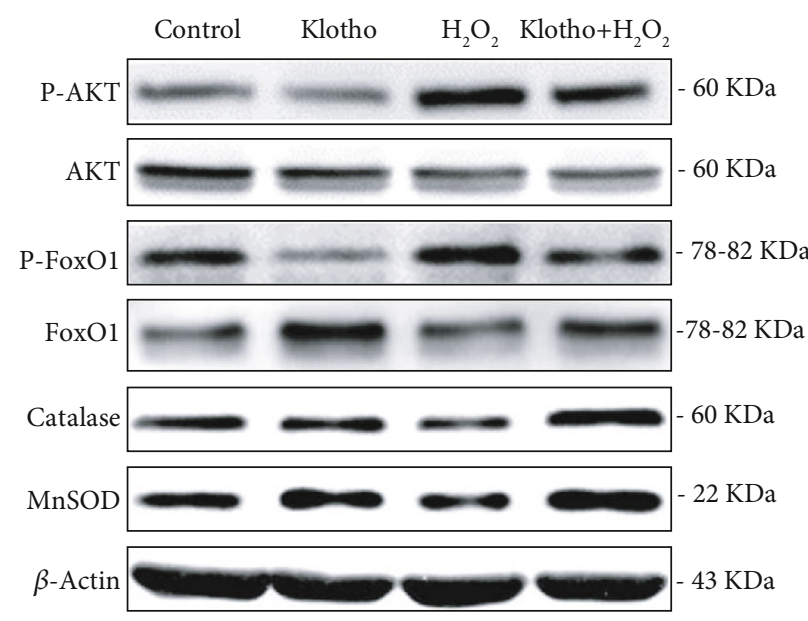

(a)

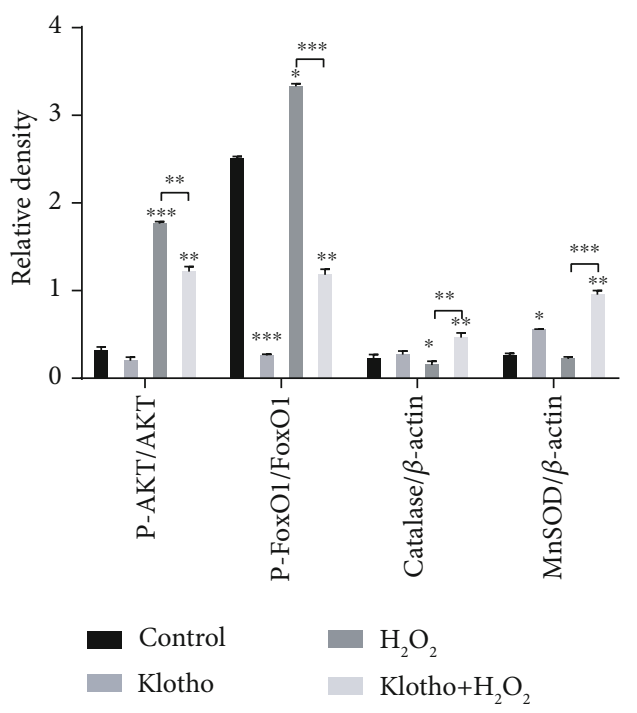

(b)

Figure 7: Klotho enhanced the Catalase and MnSOD expression by regulating the AKT/FoxO1 pathway in $\mathrm{H}_{2} \mathrm{O}_{2}$-treated hPDLSCs. Western blotting results (a) and relative density (b) of the protein expression of AKT, phosphorylated AKT (p-AKT), phosphorylated FoxO1 (p-FoxO1), FoxO1, Catalase, and MnSOD. ${ }^{*} P<0.05,{ }^{* *} P<0.01$, and ${ }^{* * *} P<0.001$.

that Klotho-treated cells had less mtDNA loss and significantly higher maximal respiration and spare respiratory capacity than did control cells (Figure 6). Spare capacity represents reserve bioenergetic capacity and is calculated as the difference between the basal and maximal OCR, indicating the ability of a cell to respond to stress [59]. In general, mitochondrial quality is controlled by interconnected systems, including elements for fighting oxygen-mediated toxicity, maintaining mitochondrial proteostasis, and regulating mitochondrial morphology, location, and number [23]. Taken together, these data support the hypothesis that Klotho administration protected the quality and bioactivity of mitochondria in hPDLSCs, and these effects may reduce $\mathrm{H}_{2} \mathrm{O}_{2}$-induced oxidative damage.

Next, we examined whether the Klotho-induced effects were mediated through the PI3K/AKT/FoxO1 pathway. The PI3K/AKT pathway has been reported to play an important role in the biological behaviour of MSCs [60]. The present study verified that Klotho increased the expression of Catalase and MnSOD. Klotho negatively mediated the $\mathrm{PI} 3 \mathrm{~K} / \mathrm{AKT}$ pathway to regulate FoxO1-mediated enzymatic antioxidant expression (Figure 7). The PI3K/AKT signalling pathway regulates FoxO through phosphorylation $[29,61$, 62]. The AKT-mediated phosphorylation of FoxO1 inhibits FoxO1 activity by promoting its nuclear exportation and proteasomal degradation [63]. FoxO1 can upregulate MnSOD and Catalase expression. However, it remains unclear whether the ability of Klotho to protect mitochondria and to confer oxidative stress resistance is entirely dependent on
Catalase and MnSOD induction. Further investigation, such as gene overexpression and knockdown of Klotho, should be performed to verify this hypothesis and molecular mechanism.

\section{Conclusion}

Thus far, the findings imply the protective effects of recombinant Klotho protein on hPDLSCs under oxidative conditions. Pretreatment with Klotho attenuated $\mathrm{H}_{2} \mathrm{O}_{2}$-induced cell damage and decreased osteogenesis by restoring the mitochondrial antioxidant system through the PI3K/AKT/FoxO1 pathway. Our study indicated that Klotho could be a promising option for protecting hPDLSCs against oxidative injuries and ensuring cell stemness for osteogenesis and tissue regeneration engineering.

\section{Data Availability}

The data used to support the findings of this study are included within the article.

\section{Conflicts of Interest}

The authors declare that there is no conflict of interest regarding the publication of this article. 


\section{Authors' Contributions}

Huan Chen and Xiaojun Huang contributed equally to this work.

\section{Acknowledgments}

This work was supported by the National Natural Science Foundation of China (No. 81530069) and the Guangdong Innovative Research Team Program (No. 2009010058).

\section{Supplementary Materials}

Figure S1: characterization of hPDLSCs. (Supplementary Materials)

\section{References}

[1] B. L. Pihlstrom, B. S. Michalowicz, and N. W. Johnson, "Periodontal diseases," Lancet, vol. 366, no. 9499, pp. 1809-1820, 2005.

[2] O. Trubiani, P. Ballerini, G. Murmura et al., "Toll-like Receptor 4 Expression, Interleukin-6,-8 and Ccl-20 Release, and NFKB Translocation in Human Periodontal Ligament Mesenchymal Stem Cells Stimulated with LPS-P. Gingivalis," European Journal of Inflammation, vol. 10, no. 1, pp. 81-89, 2012.

[3] L. J. A. Heitz-Mayfield and N. P. Lang, "Surgical and nonsurgical periodontal therapy. Learned and unlearned concepts," Periodontology 2000, vol. 62, no. 1, pp. 218-231, 2013.

[4] S. A. Khan, E. F. Kong, T. F. Meiller, and M. A. Jabra-Rizk, "Periodontal diseases: bug induced, host promoted," PLoS Pathogens, vol. 11, no. 7, p. e1004952, 2015.

[5] J. Nuñez, F. Vignoletti, R. G. Caffesse, and M. Sanz, "Cellular therapy in periodontal regeneration," Periodontology 2000, vol. 79, no. 1, pp. 107-116, 2019.

[6] R. O. Oreffo, C. Cooper, C. Mason, and M. Clements, "Mesenchymal stem cells: lineage, plasticity, and skeletal therapeutic potential," Stem Cell Reviews, vol. 1, no. 2, pp. 169-178, 2005.

[7] E. Eleuterio, O. Trubiani, M. Sulpizio et al., "Proteome of human stem cells from periodontal ligament and dental pulp," PLoS One, vol. 8, no. 8, p. e71101, 2013.

[8] O. Trubiani, J. Pizzicannella, S. Caputi et al., "Periodontal ligament stem cells: current knowledge and future perspectives," Stem Cells and Development, vol. 28, no. 15, pp. 995-1003, 2019.

[9] B. M. Seo, M. Miura, S. Gronthos et al., "Investigation of multipotent postnatal stem cells from human periodontal ligament," Lancet, vol. 364, no. 9429, pp. 149-155, 2004.

[10] F. M. Chen, H. H. Sun, H. Lu, and Q. Yu, "Stem cell-delivery therapeutics for periodontal tissue regeneration," Biomaterials, vol. 33, no. 27, pp. 6320-6344, 2012.

[11] J. Y. Park, S. H. Jeon, and P. H. Choung, "Efficacy of periodontal stem cell transplantation in the treatment of advanced periodontitis," Cell Transplantation, vol. 20, no. 2, pp. 271-285, 2011.

[12] Q. Ou, Y. Miao, F. Yang, X. Lin, L. M. Zhang, and Y. Wang, "Zein/gelatin/nanohydroxyapatite nanofibrous scaffolds are biocompatible and promote osteogenic differentiation of human periodontal ligament stem cells," Biomaterials Science, vol. 7, no. 5, pp. 1973-1983, 2019.
[13] F. M. Chen, L. N. Gao, B. M. Tian et al., "Treatment of periodontal intrabony defects using autologous periodontal ligament stem cells: a randomized clinical trial," Stem Cell Research \& Therapy, vol. 7, no. 1, 2016.

[14] Y. Wen, H. Yang, J. Wu et al., "COL4A2 in the tissue-specific extracellular matrix plays important role on osteogenic differentiation of periodontal ligament stem cells," Theranostics, vol. 9, no. 15, pp. 4265-4286, 2019.

[15] Y. Zheng, C. Dong, J. Yang et al., "Exosomal microRNA-155$5 p$ from PDLSCs regulated Th17/Treg balance by targeting sirtuin-1 in chronic periodontitis," Journal of Cellular Physiology, vol. 234, no. 11, pp. 20662-20674, 2019.

[16] C. Hu and L. Li, "Melatonin plays critical role in mesenchymal stem cell-based regenerative medicine in vitro and in vivo," Stem Cell Research \& Therapy, vol. 10, no. 1, 2019.

[17] A. M. Collignon, J. Lesieur, C. Vacher, C. Chaussain, and G. Y. Rochefort, "Strategies developed to induce, direct, and potentiate bone healing," Frontiers in Physiology, vol. 8, 2017.

[18] L. Danisovic, L. Oravcova, L. Krajciova et al., "Effect of longterm culture on the biological and morphological characteristics of human adipose tissue-derived stem cells," Journal of Physiology and Pharmacology, vol. 68, no. 1, pp. 149-158, 2017.

[19] J. Wu, J. Li, N. Zhang, and C. Zhang, "Stem cell-based therapies in ischemic heart diseases: a focus on aspects of microcirculation and inflammation," Basic Research in Cardiology, vol. 106, no. 3, pp. 317-324, 2011.

[20] K. B. Choo, L. Tai, K. S. Hymavathee et al., "Oxidative stressinduced premature senescence in Wharton's jelly-derived mesenchymal stem cells," International Journal of Medical Sciences, vol. 11, no. 11, pp. 1201-1207, 2014.

[21] K. Ksiazek, "A comprehensive review on mesenchymal stem cell growth and senescence," Rejuvenation Research, vol. 12, no. 2, pp. 105-116, 2009.

[22] S. Dikalov, "Cross talk between mitochondria and NADPH oxidases," Free Radical Biology \& Medicine, vol. 51, no. 7, pp. 1289-1301, 2011.

[23] J. C. Campos, T. Fernandes, L. R. G. Bechara et al., "Increased clearance of reactive aldehydes and damaged proteins in hypertension-induced compensated cardiac hypertrophy: impact of exercise training," Oxidative Medicine and Cellular Longevity, vol. 2015, Article ID 464195, 11 pages, 2015.

[24] J. St-Pierre, S. Drori, M. Uldry et al., "Suppression of reactive oxygen species and neurodegeneration by the PGC-1 transcriptional coactivators," Cell, vol. 127, no. 2, pp. 397-408, 2006.

[25] S. G. Jeong and G. W. Cho, "Accumulation of apoptosisinsensitive human bone marrow-mesenchymal stromal cells after long-term expansion," Cell Biochemistry and Function, vol. 34, no. 5, pp. 310-316, 2016.

[26] J. Lee, Y. S. Cho, H. Jung, and I. Choi, "Pharmacological regulation of oxidative stress in stem cells," Oxidative Medicine and Cellular Longevity, vol. 2018, Article ID 4081890, 13 pages, 2018.

[27] P. Venditti, L. Di Stefano, and S. Di Meo, "Mitochondrial metabolism of reactive oxygen species," Mitochondrion, vol. 13, no. 2, pp. 71-82, 2013.

[28] M. Kuro-o, Y. Matsumura, H. Aizawa et al., "Mutation of the mouse klotho gene leads to a syndrome resembling ageing," Nature, vol. 390, no. 6655, pp. 45-51, 1997. 
[29] G. D. Dalton, J. Xie, S. W. An, and C. L. Huang, "New insights into the mechanism of action of soluble Klotho," Frontiers in Endocrinology, vol. 8, 2017.

[30] S. Song and L. Y. Si, "Klotho ameliorated isoproterenolinduced pathological changes in cardiomyocytes via the regulation of oxidative stress," Life Sciences, vol. 135, pp. 118-123, 2015.

[31] M. Yamamoto, J. D. Clark, J. V. Pastor et al., "Regulation of oxidative stress by the anti-aging hormone klotho," The Journal of Biological Chemistry, vol. 280, no. 45, pp. 3802938034, 2005.

[32] A. Sahu, H. Mamiya, S. N. Shinde et al., "Age-related declines in $\alpha$-Klotho drive progenitor cell mitochondrial dysfunction and impaired muscle regeneration," Nature Communications, vol. 9, no. 1, p. 4859, 2018.

[33] P. Chuchana, A. L. Mausset-Bonnefont, M. Mathieu et al., "Secreted $\alpha$-Klotho maintains cartilage tissue homeostasis by repressing and catabolic axis," Aging, vol. 10, no. 6, pp. 1442-1453, 2018.

[34] Y. Guo, X. Zhuang, Z. Huang et al., "Klotho protects the heart from hyperglycemia-induced injury by inactivating ROS and NF- $\kappa \mathrm{B}$-mediated inflammation both in vitro and in vivo," Biochimica et Biophysica Acta - Molecular Basis of Disease, vol. 1864, no. 1, pp. 238-251, 2018.

[35] L. B. Xie, X. Chen, B. Chen, X. D. Wang, R. Jiang, and Y. P. Lu, "Protective effect of bone marrow mesenchymal stem cells modified with klotho on renal ischemia-reperfusion injury," Renal Failure, vol. 41, no. 1, pp. 175-182, 2019.

[36] R. Bi, A. M. Zhang, W. Zhang et al., "The acquisition of an inheritable 50-bp deletion in the human mtDNA control region does not affect the mtDNA copy number in peripheral blood cells," Human Mutation, vol. 31, no. 5, pp. 538-543, 2010.

[37] C. Giorgi, M. Bonora, S. Missiroli et al., "Alterations in mitochondrial and endoplasmic reticulum signaling by p53 mutants," Frontiers in Oncology, vol. 6, 2016.

[38] S. Singh, R. D. Moirangthem, A. Vaidya, S. Jalnapurkar, L. Limaye, and V. Kale, "AKT signaling prevailing in mesenchymal stromal cells modulates the functionality of hematopoietic stem cells via intercellular communication," Stem Cells, vol. 34, no. 9, pp. 2354-2367, 2016.

[39] L. Liao, X. Su, X. Yang et al., "TNF- $\alpha$ inhibits FoxO1 by upregulating miR-705 to aggravate oxidative damage in bone marrow-derived mesenchymal stem cells during osteoporosis," Stem Cells, vol. 34, no. 4, pp. 1054-1067, 2016.

[40] R. Langer and J. P. Vacanti, "Tissue engineering," Science, vol. 260, no. 5110, pp. 920-926, 1993.

[41] J. Kobayashi, A. Kikuchi, T. Aoyagi, and T. Okano, "Cell sheet tissue engineering: cell sheet preparation, harvesting/manipulation, and transplantation," Journal of Biomedical Materials Research. Part A, vol. 107, no. 5, pp. 955-967, 2019.

[42] R. A. Denu and P. Hematti, "Effects of oxidative stress on mesenchymal stem cell biology," Oxidative Medicine and Cellular Longevity, vol. 2016, Article ID 2989076, 9 pages, 2016.

[43] L. Ferrucci and E. Fabbri, "Inflammageing: chronic inflammation in ageing, cardiovascular disease, and frailty," Nature Reviews Cardiology, vol. 15, no. 9, pp. 505-522, 2018.

[44] H. Liu, F. Anders, S. Thanos et al., "Hydrogen sulfide protects retinal ganglion cells against glaucomatous injury in vitro and in vivo," Investigative Ophthalmology \& Visual Science, vol. 58, no. 12, pp. 5129-5141, 2017.
[45] J. G. Farías, V. M. Molina, R. A. Carrasco et al., “Antioxidant therapeutic strategies for cardiovascular conditions associated with oxidative stress," Nutrients, vol. 9, no. 9, p. 966, 2017.

[46] R. Vergara, F. Parada, S. Rubio, and F. J. Pérez, "Hypoxia induces $\mathrm{H}_{2} \mathrm{O}_{2}$ production and activates antioxidant defence system in grapevine buds through mediation of $\mathrm{H}_{2} \mathrm{O}_{2}$ and ethylene," Journal of Experimental Botany, vol. 63, no. 11, pp. 4123-4131, 2012.

[47] L. F. Dmitriev and V. N. Titov, "Lipid peroxidation in relation to ageing and the role of endogenous aldehydes in diabetes and other age-related diseases," Ageing Research Reviews, vol. 9, no. 2, pp. 200-210, 2010.

[48] M. M. Gaschler and B. R. Stockwell, "Lipid peroxidation in cell death," Biochemical and Biophysical Research Communications, vol. 482, no. 3, pp. 419-425, 2017.

[49] C. Hsia, P. Ravikumar, and J. Ye, "Acute lung injury complicating acute kidney injury: a model of endogenous $\alpha$ Klotho deficiency and distant organ dysfunction," Bone, vol. 100, pp. 100-109, 2017.

[50] D. Tsikas, "Assessment of lipid peroxidation by measuring malondialdehyde (MDA) and relatives in biological samples: analytical and biological challenges," Analytical Biochemistry, vol. 524, pp. 13-30, 2017.

[51] H. Wang, Y. Chen, N. Zhai et al., "Ochratoxin A-induced apoptosis of IPEC-J2 cells through ROS-mediated mitochondrial permeability transition pore opening pathway," Journal of Agricultural and Food Chemistry, vol. 65, no. 48, pp. 1063010637, 2017.

[52] H. H. Szeto, "First-in-class cardiolipin-protective compound as a therapeutic agent to restore mitochondrial bioenergetics," British Journal of Pharmacology, vol. 171, no. 8, pp. 2029-2050, 2014.

[53] S. J. Kim, P. Cheresh, M. Eren et al., "Klotho, an antiaging molecule, attenuates oxidant-induced alveolar epithelial cell mtDNA damage and apoptosis," American Journal of Physiology. Lung Cellular and Molecular Physiology, vol. 313, no. 1, pp. L16-L26, 2017.

[54] S. W. Lim, L. Jin, K. Luo et al., "Klotho enhances FoxO3mediated manganese superoxide dismutase expression by negatively regulating PI3K/AKT pathway during tacrolimusinduced oxidative stress," Cell Death \& Disease, vol. 8, no. 8, p. e2972, 2017.

[55] F. Shang, S. Liu, L. Ming et al., "Human umbilical cord MSCs as new cell sources for promoting periodontal regeneration in inflammatory periodontal defect," Theranostics, vol. 7, no. 18, pp. 4370-4382, 2017.

[56] J. Zhou, F. Wang, Y. Ma, and F. Wei, "Vitamin D3 contributes to enhanced osteogenic differentiation of MSCs under oxidative stress condition via activating the endogenous antioxidant system," Osteoporosis International, vol. 29, no. 8, pp. 19171926, 2018.

[57] L. Zhang, X. Gan, Y. He, Z. Zhu, J. Zhu, and H. Yu, “Drp1dependent mitochondrial fission mediates osteogenic dysfunction in inflammation through elevated production of reactive oxygen species," PLoS One, vol. 12, no. 4, p. e0175262, 2017.

[58] C. T. Chen, Y. R. Shih, T. K. Kuo, O. K. Lee, and Y. H. Wei, "Coordinated changes of mitochondrial biogenesis and antioxidant enzymes during osteogenic differentiation of human mesenchymal stem cells," Stem Cells, vol. 26, no. 4, pp. 960 968, 2008. 
[59] M. B. de Moura and B. Van Houten, "Bioenergetic analysis of intact mammalian cells using the Seahorse XF24 Extracellular Flux analyzer and a luciferase ATP assay," Methods in Molecular Biology, vol. 1105, pp. 589-602, 2014.

[60] H. Zhou, D. Li, C. Shi et al., "Effects of Exendin-4 on bone marrow mesenchymal stem cell proliferation, migration and apoptosis_in vitro_,"Scientific Reports, vol. 5, no. 1, 2015.

[61] H. Rakugi, N. Matsukawa, K. Ishikawa et al., "Anti-oxidative effect of Klotho on endothelial cells through cAMP activation," Endocrine, vol. 31, no. 1, pp. 82-87, 2007.

[62] D. H. Kim, M. H. Park, S. Ha et al., "Anti-inflammatory action of $\beta$-hydroxybutyrate via modulation of PGC- $1 \alpha$ and FoxO1, mimicking calorie restriction," Aging (Albany NY), vol. 11, no. 4, pp. 1283-1304, 2019.

[63] M. Saline, L. Badertscher, M. Wolter et al., "AMPK and AKT protein kinases hierarchically phosphorylate the N-terminus of the FOXO1 transcription factor, modulating interactions with 14-3-3 proteins," The Journal of Biological Chemistry, vol. 294, no. 35, pp. 13106-13116, 2019. 


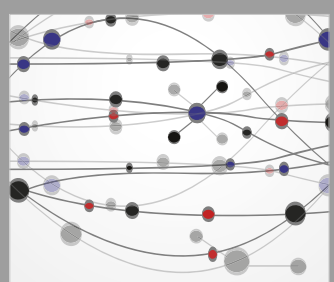

The Scientific World Journal
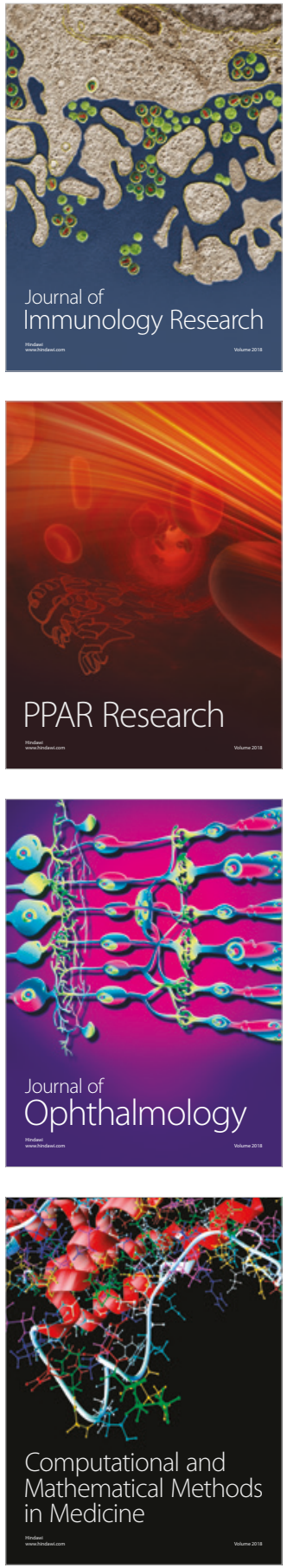

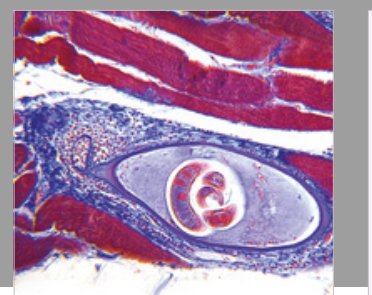

Gastroenterology Research and Practice

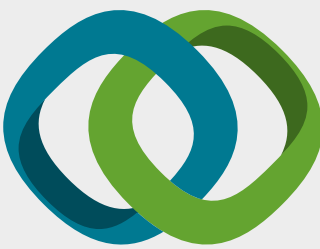

\section{Hindawi}

Submit your manuscripts at

www.hindawi.com
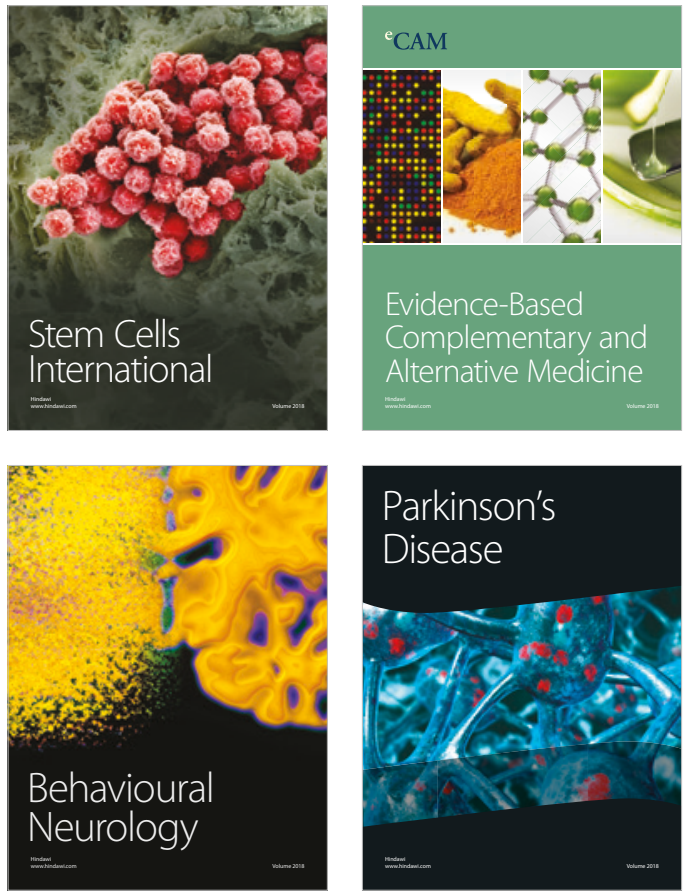

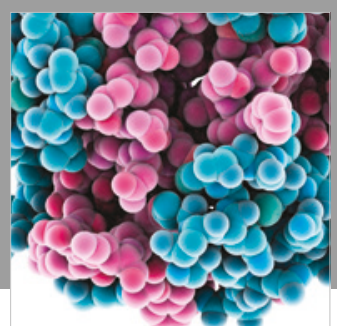

ournal of

Diabetes Research

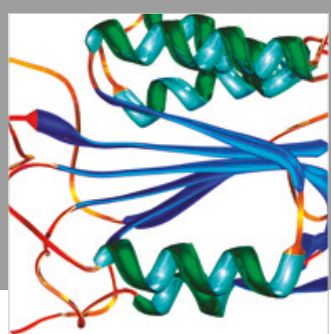

Disease Markers
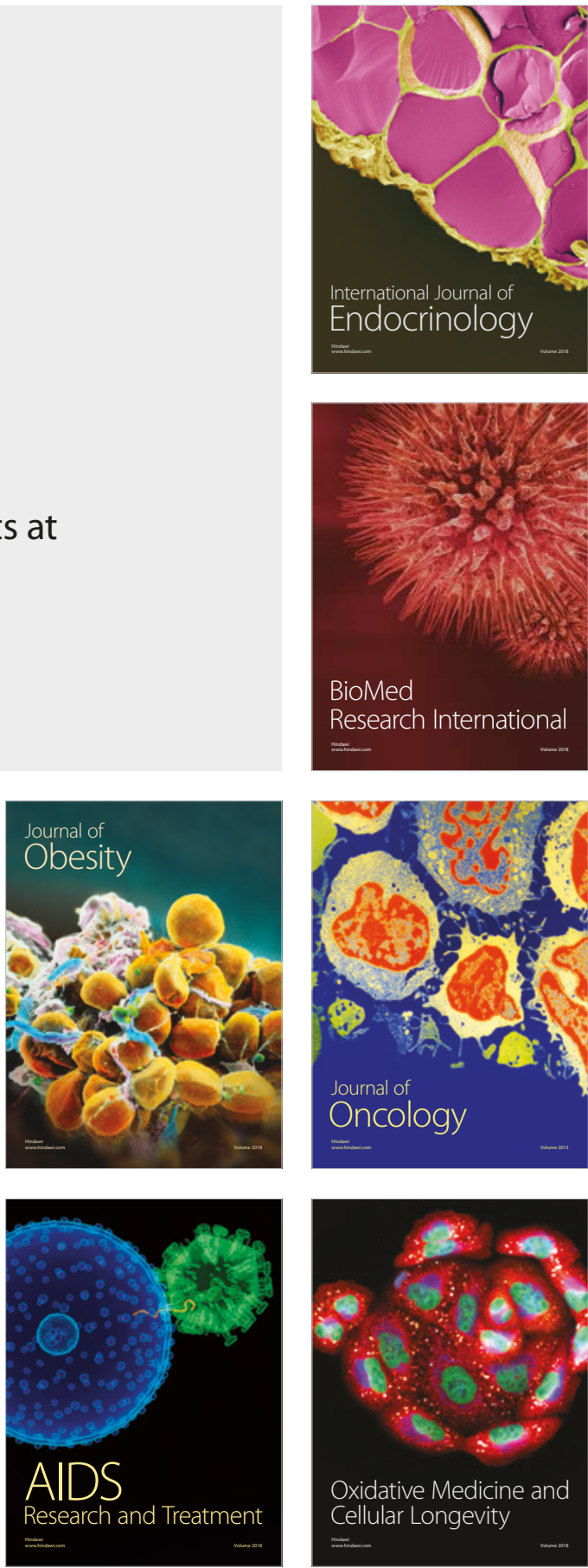